\title{
Acute Kidney Injury in Children and Its Potential Consequences in Adulthood
}

\author{
Stuart L. Goldstein \\ Center for Acute Care Nephrology, Division of Nephrology and Hypertension, The Heart Institute, \\ Cincinnati Children's Hospital Medical Center, Cincinnati, Ohio, USA
}

\section{Key Words}

Acute kidney injury $\cdot$ Chronic kidney disease $\cdot$ Pediatrics

\begin{abstract}
While emerging evidence indicates that the incidence of both acute kidney injury (AKI) and chronic kidney disease (CKD) in children is rising, and the etiologies are dramatically changing, relatively little is currently known regarding the potential for transition from AKI to CKD. In both situations, early intervention can significantly improve the dismal prognosis. Fortunately, recent data have validated a multidimensional AKI classification system for children, and led to the investigation of the chronic kidney sequelae in many pediatric populations with $\mathrm{AKI}$, or at risk for AKI (children with hemolytic uremic syndrome, neonates or those exposed to repeated nephrotoxic medications). The purpose of this article is to review the changing epidemiology of pediatric AKI and its potential effect on the development of CKD in children.
\end{abstract}

Copyright $\odot 2012$ S. Karger AG, Basel

\section{Introduction}

Advancement in the care of children with kidney disease has led to dramatic improvements in patient survival. However, most pediatric kidney epidemiological stud- ies have focused on long-term outcomes in children with end-stage renal disease (ESRD) $[1,2]$. Care for the critically ill child with acute kidney injury (AKI) has improved greatly, with survival rates reaching $60-70 \%$, even for children who require renal replacement therapy (RRT) $[3,4]$. Recently, researchers have recognized that episodes of AKI can lead to more rapid progression to chronic kidney disease (CKD) in adult patients; the 2009 United States Renal Data System report revealed that adults with an AKI episode during hospitalization have an approximately tenfold greater risk of progressing to ESRD within 6-12 months than patients who have not experienced AKI [5]. No similar data exist in children; however, with the potential for survival for many decades after an AKI episode, identification of children at risk for CKD progression after AKI is especially important. The purpose of this article is to review the changing epidemiology of pediatric AKI and its potential effect on the development of CKD in children.

\section{Epidemiology of Pediatric AKI}

The epidemiology of pediatric acute kidney injury (pAKI) has mainly been studied in acutely ill hospitalized patients, since nonoliguric forms of pAKI may be self-limited and go undetected in the outpatient setting. While

\section{KARGER \\ Fax +4161306 1234 E-Mail karger@karger.ch} www.karger.com
() 2012 S. Karger AG, Basel 0253-5068/12/0333-0131\$38.00/0

Accessible online at: www.karger.com/bpu
Stuart L. Goldstein, MD

Cincinnati Children's Hospital Medical Center

3333 Burnet Avenue, MLC 7022

Cincinnati, OH 45206 (USA)

Tel. +1 513636 2209, E-Mail stuart.goldstein@cchmc.org 
Table 1. Shift in pAKI epidemiology

\begin{tabular}{llll}
\hline Author & Time span & Cohort & AKI cause \\
\hline Williams et al. [8], 2002 & $1978-1998$ & all hospital & $\begin{array}{l}\text { 1978-88: HUS 38\%, oncology 8\% } \\
\text { 1988-98: HUS 22\%, oncology 17\% }\end{array}$ \\
\hline Hui-Stickle et al. [4], 2005 & $1999-2001$ & all hospital & $\begin{array}{l}\text { ischemic 21\% } \\
\text { nephrotoxins 16\% } \\
\text { primary renal 7\% }\end{array}$ \\
& & & $\begin{array}{l}\text { pneumonia 33\% } \\
\text { Akcan-Arikan et al. [20], 2007 }\end{array}$ \\
& $2005-2006$ & pediatric intensive care unit 27\% \\
& & & cardiogenic 10\% \\
\hline Ball and Kara [10], 2008 & $2001-2006$ & $\begin{array}{l}\text { pediatric intensive care unit } \\
\text { receiving RRT }\end{array}$ & $\begin{array}{l}\text { cardiogenic 58\% } \\
\text { HUS 17\% } \\
\text { sepsis 13\% }\end{array}$ \\
\hline
\end{tabular}

multicenter data do not exist, single center studies from the 1980s and 1990s report hemolytic uremic syndrome (HUS), other primary renal causes, sepsis, and burns as the most prevalent causes leading to pAKI [6,7]. More recent pediatric data $[4,8-10]$ reveal a dramatic shift in the epidemiology of pAKI (table 1), with the most common causes being renal ischemia (often after cardiopulmonary bypass surgery [10]), nephrotoxin use, and sepsis; thus, pAKI more often develops in hospitalized children as a result of another systemic illness or its treatment and not from primary kidney disease. pAKI epidemiological study has intensified over recent years, likely as a result of more widespread provision of acute RRT modalities to critically ill children [11]. Hospital and pediatric intensive care unit-acquired pAKI rates appear to have increased more than ninefold from the 1980s through 2004, likely due to increased use of more invasive management and higher illness severity of critically ill children [9].

Until recently, pAKI studies suffered from a lack of a standardized definition, with differing definitions from varying increases in serum creatinine or decreases in urine output, to RRT provision. The incidence of the most severe forms of pAKI, defined by dialysis requirement, ranges from 1 to $2 \%$ of all critically ill children [9, 12]. In children undergoing cardiopulmonary bypass, the incidence of $\mathrm{AKI}$ is in the range of $10-50 \%$, depending on the definition used [13-15]. In addition, a long-held concept that critically ill patients died 'with' and not 'from' AKI has recently been challenged [16]. Even small increases in serum creatinine, much less than would be considered indicative of the need for RRT, are now recognized to contribute to poor outcomes. Chertow et al. [17] demonstrated that increases in serum creatinine of 0.3 $\mathrm{mg} / \mathrm{dl}$ were associated with increased adult patient mortality, even when outcome was controlled for significant patient comorbidity. Similar results were noted in pediatric patients with acute decompensated heart failure; patients with a $0.3-\mathrm{mg} / \mathrm{dl}$ or greater serum creatinine rise demonstrated a sevenfold increased mortality risk [18]. These studies highlight the need for more refined AKI definitions and to focus on earlier detection of AKI.

In 2004, a standardized AKI consensus definition was proposed by the Acute Dialysis Quality Initiative: the RIFLE criteria (risk, injury, failure, loss, ESRD), which based AKI diagnosis and severity on changes in serum creatinine from baseline and/or degree and duration of oliguria [19]. The adult-derived RIFLE definition was modified, and then applied and validated in pediatric patients and renamed the pediatric RIFLE (pRIFLE) criteria. pRIFLE stratifies AKI from mild (RIFLE-R, 'risk') to severe (RIFLE-F, 'failure') based on changes in serum creatinine or estimated creatinine clearance and urine output (table 2). The rationale for using estimated creatinine clearance instead of absolute changes in serum creatinine emanated from the widely varying body mass seen in the pediatric population of neonates to young adults, leading to different levels of normal baseline serum creatinine.

The first study which defined AKI using the pRIFLE criteria found that AKI occurred in $82 \%$ of critically ill children admitted to a pediatric intensive care unit who received invasive mechanical ventilation and at least one vasoactive medication [20]. Similar to adult studies [21, 22], worsening pAKI defined by pRIFLE criteria was an independent risk factor for mortality and increased hos- 
Table 2. Pediatric modified RIFLE criteria [20]

\begin{tabular}{lll}
\hline & Pediatric modified RIFLE criteria & \\
\cline { 2 - 3 } & eCCl by Schwartz formula & urine output \\
\hline Risk & eCCl decrease by $25 \%$ & $<0.5 \mathrm{ml} / \mathrm{kg} / \mathrm{h}$ for $8 \mathrm{~h}$ \\
Injury & eCCl decrease by $50 \%$ & $<0.5 \mathrm{ml} / \mathrm{kg} / \mathrm{h}$ for $16 \mathrm{~h}$ \\
Failure & eCCl decrease by $75 \%$ or eCCl $<35 \mathrm{ml} / \mathrm{min} / 1.73 \mathrm{~m}^{2}$ & $<0.3 \mathrm{ml} / \mathrm{kg} / \mathrm{h}$ for $24 \mathrm{~h}$ or anuric for $12 \mathrm{~h}$ \\
Loss & persistent failure $>4$ weeks & \\
End stage & ESRD (persistent failure $>3$ months) & \\
\hline
\end{tabular}

$\mathrm{eCCl}=$ Estimated creatinine clearance.

pital length of stay. Subsequent reports confirm the utility of the pRIFLE criteria to stratify pAKI severity, highlighting the potential association between pAKI severity and patient outcome [23-25]. Additionally, pRIFLE strata have served as useful outcome measures in terms of the validation of novel urinary pAKI biomarkers to predict pAKI severity in critically ill children [26,27]. The adult RIFLE criteria have been modified by the Acute Kidney Injury Network (AKIN) to specify a $>0.3-\mathrm{mg} / \mathrm{dl}$ serum creatinine rise in $48 \mathrm{~h}$ denotes mild AKI (AKIN stage 1 or RIFLE-R) [28]. The AKIN criteria were developed with input from pediatric nephrologists; preliminary comparisons show that pRIFLE and AKIN lead to similar diagnostic AKI rates, with a potential difference in AKI severity distribution $[25,29]$.

Few prospective studies exist to accurately assess risk factors for pAKI development. Most pAKI studies assess patients who have already developed AKI, examining the variables common among the pAKI population of interest. However, such studies do not examine a control population with similar exposure risks to determine the true risk associated with each clinical variable. It is clear, though, that worsening illness severity in itself is a risk factor for developing AKI. The critically ill patient who is intubated and receiving vasoactive medications should prompt early vigilance for AKI occurrence. pAKI incidence is extremely high (82\%) in more severely ill patients [20] compared to all patients admitted to the pediatric intensive care unit (4.5\%) [12].

The recent research into pAKI epidemiology has begun to yield new and important data. Nonetheless, further prospective epidemiologic research utilizing a common definition, with a detailed description of the particular population studied, will be crucial to understanding the true incidence of mild-to-severe AKI in patient populations with different underlying diagnoses. In fact, a recent publication proposed the concept of a 'renal angina equivalent' to prompt investigation into the presence and causes of AKI, much as chest pain and associated signs and symptoms prompt evaluation for acute coronary syndrome and myocardial infarction [30]. The pediatric metrics for renal angina include invasive mechanical ventilation, vasoactive substance use, history of recent stem cell transplantation, and/or the development of relative fluid overload. In fact, given that data from over 700 children in six studies demonstrated a consistent association between intensive care unit fluid overload of $>10-20 \%$ at continuous RRT initiation and mortality in children, fluid overload may be a strong indicator of pAKI. In the very near future, we will be able to clinically stratify patients at risk for pAKI using the renal angina concept to direct and optimize the use of novel pAKI biomarkers to detect pAKI early (i.e. prior to a rise in serum creatinine or decrease in urine output), and hopefully test interventions to prevent or mitigate the effects of AKI. Based on the clinical situation, pAKI biomarkers could be incorporated into a further refined pRIFLE AKI definition.

\section{Epidemiology of Pediatric CKD}

Pediatric CKD epidemiological data can be derived from large national or multinational database registries including from the European Dialysis and Transplantation Association-European Renal Association, United States Renal Data Renal Data System, Canadian Organ Replacement Register, Registry of the Japanese Society for Dialysis Therapy, Australia and New Zealand Dialysis and Transplant Registry, and North American Pediatric Renal Trials and Collaborative Studies (NAPRTCS) [31]. Each registry differs with respect to the detail of the data obtained regarding patient age stratification, specific dis- 
ease categories leading to CKD available for selection, and whether the data come from government mandated versus voluntary enrollment.

While a detailed description of each registry is beyond the scope of this paper, the focus of most of the registries is placed upon patients with ESRD. The NAPRTCS database, which is a voluntary registry from North American pediatric centers, established a separate chronic renal insufficiency arm in 1994 [32]. The 2008 NAPRTCS Annual Report (www.naprtcs.org) contains data from 7,037 children $<20$ years of age with chronic renal insufficiency, defined as an estimated creatinine clearance of $<75 \mathrm{ml} /$ $\min / 1.73 \mathrm{~m}^{2}$. The leading primary diagnoses responsible for chronic renal insufficiency mirror those reported for ESRD and include both anatomical/hereditary lesions such as dysplasia, reflux nephropathy, and obstructive uropathy, as well as chronic glomerulopathies. Of note, no specific categories for AKI or cortical necrosis are listed, which may result from a lack of recognition of AKI as a primary diagnosis leading to CKD. Long-term longitudinal study of pAKI survivors is warranted to determine if pAKI will become a more prevalent cause of CKD.

Future insights into the epidemiology of pediatric CKD will be gained from the ongoing North American Chronic Kidney Disease in Children trial [33]. This represents a prospective cohort study of 540 children aged 1-16 years with an estimated GFR between 30 and $75 \mathrm{ml} /$ $\min / 1.73 \mathrm{~m}^{2}$, established to identify novel risk factors for CKD progression. More importantly, the Chronic Kidney Disease in Children cohort study has recently reassessed old GFR estimation equations [34] and validated a new GFR estimation equation used to classify children with different stages of CKD [35]. This new formula, i.e. estimated GFR $=0.413 \cdot($ height $($ in $\mathrm{cm}$ )/serum creatinine), has been validated prospectively with iohexol renal scans and will likely identify many more children with CKD as the older formula overestimated GFR due to its study in mostly children with normal GFR or ESRD. Those studies yielded a higher coefficient $(0.45-0.75$, based on patient age and gender) than the newer validated formula. As with AKI, future research may lead us to define CKD by means other than serum creatinine, such as cystatin $\mathrm{C}$ $[36,37]$ or other urinary biomarkers $[13,38-41]$.

\section{Does Pediatric AKI Lead to CKD?}

The long-term sequelae of pAKI have only been the subject of recent investigation, since, as noted above, no systematic assessment of pAKI survivors has been un- dertaken. Ball and Kara [10] found that $40 \%$ of surviving pAKI patients who received acute RRT had signs of a kidney abnormality (decreased GFR, hypertension, hematuria, or proteinuria) at the time of hospital discharge, and patients with primary renal disease-associated pAKI had a higher rate of renal dysfunction at hospital discharge. Hui-Stickle et al. [4] demonstrated that $34 \%$ of 176 children had either reduced kidney function or were dialysis dependent upon discharge from a tertiary center after a pAKI episode. Askenazi et al. [42] followed this cohort for 3-5 years and found patient survival to be $56.8 \%$, with the majority of mortality occurring within 2 years of the pAKI episode. Sixteen children progressed to ESRD, with the majority (91\%) having an underlying renal or urological disease. In addition, 17 of 29 patients studied in a follow-up clinic visit demonstrated evidence of CKD, manifesting as hyperfiltration, reduced kidney function, hypertension, or microalbuminuria. More concerning was that the majority of the children with CKD had more than one sign or symptom of CKD, yet only six of the children were actively being followed by a pediatric nephrologist. This early small-scale study should prompt the pediatric nephrology community to perform systematic longitudinal evaluations for pediatric CKD in children who survive a pAKI episode. Currently, the Translational Research Investigating Biomarker Endpoints for Acute Kidney Injury study consortium has formed an ancillary study group, the Assessment, Serial Evaluation, and Subsequent Sequelae in Acute Kidney Injury consortium, which will look at long-term kidney outcomes in patients with bypass-associated AKI, including children from two pediatric centers.

Other large cohort studies lend additional insight into the potential for pediatric AKI to lead to CKD. Recent meta-analyses and single-center data have been reported to assess the risk for CKD development in children suffering from HUS, pediatric cancer survivors, and premature birth. Garg et al. [43] performed an extensive meta-analysis of the long-term consequences of HUS. While this study did not directly address whether or not AKI led to CKD, the investigators did assess for an association between initial serum creatinine levels, RRT provision (presumably for AKI), and long-term renal outcomes. After screening nearly 3,400 articles, the authors included 49 studies comprising 3,476 patients for review.

The analyses primary aims were determining the incidence of, and risk factors for, the development of death, ESRD, or CKD (defined as an estimated GFR $<80 \mathrm{ml}$ / 
$\min / 1.73 \mathrm{~m}^{2}$ ). Secondary renal endpoints included proteinuria and hypertension. The death and ESRD rates from the individual studies ranged from $0-23$ and $0-17 \%$, respectively, with a combined death-ESRD rate of $12 \%$ (95\% CI: $10-15)$. The pooled estimates for CKD development were $8 \%$ (95\% CI: $5-11$ ) for $60-80 \mathrm{ml} /$ $\min / 1.73 \mathrm{~m}^{2}, 6 \%$ (95\% CI: 3-8) for GFR 30-59 ml/ $\mathrm{min} / 1.73 \mathrm{~m}^{2}$, and $1.8 \%$ (95\% CI: $0.8-3$ ) for GFR $5-29 \mathrm{ml} /$ $\mathrm{min} / 1.73 \mathrm{~m}^{2}$. In addition, the pooled rates for proteinuria and hypertension were 15\% (95\% CI: 10-20) and 10\% (95\% CI: 8-12), respectively. The combined renal sequelae estimated was $25 \%$ (95\% CI: 20-30), indicating that fully one quarter of HUS survivors are at risk for long-term kidney damage. Severity of clinical illness, defined as central nervous system symptoms, need for RRT, higher white blood cell counts, or initial serum creatinine concentrations were all associated with increased likelihood of death or long-term kidney sequelae. An important finding of this study was the observation of the development of long-term kidney sequelae in $8-61 \%$ of patients who had return of normal GFR and/ or loss of proteinuria after the acute HUS episode. Thus, as with the previous study of Askenazi et al. [42], longterm follow-up for at risk patients is warranted, even for those patients who seem to have 'renal recovery' after an acute HUS episode.

Premature neonates provide an informative pediatric population for study since they can be exposed to numerous nephrotoxic insults (e.g. sepsis, nephrotoxic medications) before nephrogenesis is complete. White et al. [44] recently performed a meta-analysis of studies examining low birth weight infants and CKD development later in life. After reviewing 1,600 studies, they were able to include 32 papers comprising 17 case-control or cohort studies (46,249 patients) and 1 genetic record linkage study. Low birth weight, defined as birth weight between 1,500 and 2,500 g, imparted a $70 \%$ risk of developing CKD later in life (HR: 1.73, 95\% CI: 1.44-2.08) and an $80 \%$ greater risk of developing albuminuria later in life (HR: 1.81, 95\% CI: 1.19-2.77) compared to normal birth weight infants.

Pediatric cancer survivors also serve as an informative cohort for long-term CKD development given the dramatic improvement in cancer treatment outcomes and their potential exposure to multiple nephrotoxic medications and insults (e.g. sepsis-induced AKI). Pediatric stem cell transplant (SCT) recipients seem to be at a particularly high risk of developing AKI, with reposted rates of 11-21\% using a pRIFLE-I equivalent (serum creatinine doubling) for an AKI definition [45-48]. As a result, the
SCT cohort has been followed for the development of CKD [47, 49, 50]. Gronroos et al. [49] reported the 7-year renal follow-up of 187 children with normal GFR before receiving SCT and found renal impairment in $41 \%$ of patients at 1 year, in $31 \%$ at 3 years, and in $11 \%$ at 7 years after SCT. Hingorani et al. [47] reported long-term kidney outcomes in 1,635 SCT recipients (279 children) with normal GFR prior to SCT and demonstrated a 23\% CKD rate with a median time to CKD of 191 days (CKD was defined as a GFR $<60 \mathrm{ml} / \mathrm{min} / 1.73 \mathrm{~m}^{2}$ ). The CKD rate in children was $4 \%$. Of note, AKI for the whole cohort was independently associated with an increased risk of CKD development (HR: 1.7, 95\% CI: 1.3-2.1), although pediatric patients were not analyzed separately for CKD risk factors. Finally, Frisk et al. [50] demonstrated a 27\% CKD rate in children 10 years after SCT who received total body irradiation.

Multiple studies have assessed for an association between nephrotoxic chemotherapeutic medication administration and chronic kidney injury in pediatric cancer patients. One study summarized the late renal effects of childhood cancer of the Children's Oncology Group, a multicenter clinical consortium, to develop guidelines for kidney-related health screening of pediatric cancer survivors [51]. This review identified that the following exposures should necessitate initial screening for hypertension, proteinuria, abnormal GFR, and electrolyte abnormalities: radiation therapy, ifosfamide, nephrectomy, methotrexate, and cisplatin/carboplatin. The recommendations did not specify the timing of initial evaluation. After the baseline evaluation, the authors recommended annual blood pressure measurement and urinalysis, with an annual assessment for hydroceles in males who have undergone nephrectomy.

More recently, two studies have specifically evaluated long-term renal function in large cohorts of pediatric long-term cancer survivors $[52,53]$. Arjmandi-Rafsanjani et al. [52] evaluated 108 children in a single Iranian center and observed a $25.2 \%$ rate of renal toxicity, including tubular disorders and hypertension. Of note, $7.5 \%$ of patients had an estimated GFR $<90 \mathrm{ml} / \mathrm{min} / 1.73 \mathrm{~m}^{2}$. We performed a similar study in 150 children to assess for CKD and chronic kidney injury, manifested by abnormal estimated GFR, proteinuria, microalbuminuria, hypertension, and/or tubulopathy [53]. We observed a high $\mathrm{CKD} /$ chronic kidney injury rate: $71 \%$ had at least 1 sign, $22 \%$ had 2 signs, 2 patients had 3 signs of CKD/injury, respectively. Nearly half of the patients had signs of CKD with an elevated or decreased estimated GFR. 


\section{Conclusions and Future Directions}

The incidence of both AKI and CKD is rising and reaching epidemic proportions. In both situations, early intervention can significantly improve the dismal prognosis. Pediatric primary care practitioners have not been as accustomed to screening for CKD as their internal medicine counterparts since $\mathrm{CKD}$ is a relatively rare disorder in children. However, as children with complex systemic disease, prematurity, or other severe acute primary kidney disease now survive AKI episodes, their risk for CKD development is becoming evident. While most of the late-effects pediatric chronic disease literature focuses on pediatric cancer survivors, we expect that children surviving sickle cell disease, cystic fibrosis, cardiac disease, and rheumatologic disease are also at risk for repeated acute renal insults that could lead to CKD. The clinical situations reviewed in this article support a practice of long-term follow-up of any child who has a severe AKI episode.

\section{References}

-1 McDonald SP, Craig JC: Long-term survival of children with end-stage renal disease. $\mathrm{N}$ Engl J Med 2004;350:2654-2662.

$\checkmark 2$ Chavers BM, Li S, Collins AJ, Herzog CA: Cardiovascular disease in pediatric chronic dialysis patients. Kidney Int 2002;62:648653.

-3 Symons JM, et al: Demographic characteristics of pediatric continuous renal replacement therapy: a report of the prospective pediatric continuous renal replacement therapy registry. Clin J Am Soc Nephrol 2007;2: 732-738.

4 Hui-Stickle S, Brewer ED, Goldstein SL: Pediatric ARF epidemiology at a tertiary care center from 1999 to 2001. Am J Kidney Dis 2005;45:96-101.

5 Collins AJ, et al: United States Renal Data System 2008 Annual Data Report. Am J Kidney Dis 2009;53:S1-S374.

$\checkmark 6$ Andreoli SP: Acute renal failure. Curr Opin Pediatr 2002;14:183-188.

$\checkmark 7$ Flynn JT: Choice of dialysis modality for management of pediatric acute renal failure. Pediatr Nephrol 2002;17:61-69.

8 Williams DM, Sreedhar SS, Mickell JJ, Chan JC: Acute kidney failure: a pediatric experience over 20 years. Arch Pediatr Adolesc Med 2002;156:893-900.

$\checkmark 9$ Vachvanichsanong P, Dissaneewate P, Lim A, McNeil E: Childhood acute renal failure: 22-year experience in a university hospital in southern Thailand. Pediatrics 2006; 118:e786-e791.

$\checkmark 10$ Ball EF, Kara T: Epidemiology and outcome of acute kidney injury in New Zealand children. J Paediatr Child Health 2008;44:642646.

$>11$ Warady BA, Bunchman T: Dialysis therapy for children with acute renal failure: survey results. Pediatr Nephrol 2000;15:11-13.

12 Bailey D, et al: Risk factors of acute renal failure in critically ill children: a prospective descriptive epidemiological study. Pediatr Crit Care Med 2007;8:29-35.

13 Mishra J, et al: Neutrophil gelatinase-associated lipocalin (NGAL) as a biomarker for acute renal injury after cardiac surgery. Lancet 2005;365:1231-1238.

14 Dent CL, et al: Plasma neutrophil gelatinaseassociated lipocalin predicts acute kidney injury, morbidity and mortality after pediatric cardiac surgery: a prospective uncontrolled cohort study. Crit Care 2007;11:R127.

15 Bennett $M$, et al: Urine NGAL predicts severity of acute kidney injury after cardiac surgery: a prospective study. Clin J Am Soc Nephrol 2008;3:665-673.

-16 Kellum JA, Angus DC: Patients are dying of acute renal failure. Crit Care Med 2002;30: 2156-2157.

17 Chertow GM, Burdick E, Honour M, Bonventre JV, Bates DW: Acute kidney injury, mortality, length of stay, and costs in hospitalized patients. J Am Soc Nephrol 2005;16:3365-3370.

18 Price JF, et al: Worsening renal function in children hospitalized with decompensated heart failure: evidence for a pediatric cardiorenal syndrome? Pediatr Crit Care Med 2008;9:279-284.

19 Bellomo R, Ronco C, Kellum JA, Mehta RL, Palevsky P: Acute renal failure - definition, outcome measures, animal models, fluid therapy and information technology needs: the Second International Consensus Conference of the Acute Dialysis Quality Initiative (ADQI) Group. Crit Care 2004;8:R204-R212.

20 Akcan-Arikan A, et al: Modified RIFLE criteria in critically ill children with acute kidney injury. Kidney Int 2007;71:1028-1035.

21 Hoste EA, Kellum JA: RIFLE criteria provide robust assessment of kidney dysfunction and correlate with hospital mortality. Crit Care Med 2006;34:2016-2017.

-22 Srisawat N, Hoste EE, Kellum JA: Modern classification of acute kidney injury. Blood Purif 2010;29:300-307.

-23 Plotz FB, Bouma AB, van Wijk JA, Kneyber MC, Bokenkamp A: Pediatric acute kidney injury in the ICU: an independent evaluation of pRIFLE criteria. Intensive Care Med 2008; 34:1713-1717.

24 Palmieri T, Lavrentieva A, Greenhalgh D: An assessment of acute kidney injury with modified RIFLE criteria in pediatric patients with severe burns. Intensive Care Med 2009; 35:2125-2129.

25 Zappitelli M, et al: Ascertainment and epidemiology of acute kidney injury varies with definition interpretation. Clin J Am Soc Nephrol 2008;3:948-954.

26 Zappitelli M, et al: Urine neutrophil gelatinase-associated lipocalin is an early marker of acute kidney injury in critically ill children: a prospective cohort study. Crit Care 2007; 11:R84.

27 Washburn KK, et al: Urinary interleukin-18 is an acute kidney injury biomarker in critically ill children. Nephrol Dial Transplant 2008;23:566-572.

28 Mehta RL, et al: Acute Kidney Injury Network: report of an initiative to improve outcomes in acute kidney injury. Crit Care 2007; 11:R31.

29 Zappitelli M, Moffett BS, Hyder A, Goldstein SL: Acute kidney injury in non-critically ill children treated with aminoglycoside antibiotics in a tertiary healthcare centre: a retrospective cohort study. Nephrol Dial Transplant 2011;26:144-150.

30 Goldstein SL, Chawla LS: Renal angina. Clin J Am Soc Nephrol 2010;5:943-949.

31 D'Amico G: Comparability of the different registries on renal replacement therapy. Am J Kidney Dis 1995;25:113-118.

32 Neu AM, Ho PL, McDonald RA, Warady BA: Chronic dialysis in children and adolescents. The 2001 NAPRTCS Annual Report. Pediatr Nephrol 2002;17:656-663.

33 Furth SL, et al: Design and methods in the Chronic Kidney Disease in Children (CKiD) prospective cohort study. Clin J Am Soc Neph 2006;1:1005-1015.

34 Schwartz GJ, Brion LP, Spitzer A: The use of plasma creatinine concentration for estimating glomerular filtration rate in infants, children, and adolescents. Pediatr Clin North Am 1987;34:571-590.

-35 Schwartz GJ, et al: New equations to estimate GFR in children with CKD. J Am Soc Nephrol 2009;20:629-637. 
-36 Zappitelli M, Joseph L, Gupta IR, Bell L, Paradis G: Validation of child serum creatinine-based prediction equations for glomerular filtration rate. Pediatr Nephrol 2007;22:272-281.

- 37 Zappitelli M, et al: Derivation and validation of cystatin C-based prediction equations for GFR in children. Am J Kidney Dis 2006;48: 221-230.

38 Mitsnefes MM, et al: Serum neutrophil gelatinase-associated lipocalin as a marker of renal function in children with chronic kidney disease. Pediatr Nephrol 2007;22:101-108.

- 39 Nickolas TL, Barasch J, Devarajan P: Biomarkers in acute and chronic kidney disease. Curr Opin Nephrol Hypertens 2008;17:127132.

40 Wheeler DS, et al: Serum neutrophil gelatinase-associated lipocalin (NGAL) as a marker of acute kidney injury in critically ill children with septic shock. Crit Care Med 2008; 36:1297-1303.

- 41 Haase M, Bellomo R, Devarajan P, Schlattmann P, Haase-Fielitz A: Accuracy of neutrophil gelatinase-associated lipocalin (NGAL) in diagnosis and prognosis in acute kidney injury: a systematic review and metaanalysis. Am J Kidney Dis 2009;54:1012-1024.
42 Askenazi DJ, Feig DI, Graham NM, HuiStickle S, Goldstein SL: 3-5 year longitudinal follow-up of pediatric patients after acute renal failure. Kidney Int 2006;69:184-189.

-43 Garg AX, et al: Long-term renal prognosis of diarrhea-associated hemolytic uremic syndrome: a systematic review, meta-analysis, and meta-regression. JAMA 2003;290:13601370.

44 White SL, et al: Is low birth weight an antecedent of CKD in later life? A systematic review of observational studies. Am J Kidney Dis 2009;54:248-261.

45 Michael M, Kuehnle I, Goldstein SL: Fluid overload and acute renal failure in pediatric stem cell transplant patients. Pediatr Nephrol 2004; 19:91-95.

46 Kist-van Holthe JE, et al: Prospective study of renal insufficiency after bone marrow transplantation. Pediatr Nephrol 2002;17: 1032-1037.

47 Hingorani S, Guthrie KA, Schoch G, Weiss NS, McDonald GB: Chronic kidney disease in long-term survivors of hematopoietic cell transplant. Bone Marrow Transplant 2007; 39:223-229.
48 Van Why SK, Friedman AL, Wei LJ, Hong R: Renal insufficiency after bone marrow transplantation in children. Bone Marrow Transplant 1991;7:383-388.

49 Gronroos $\mathrm{MH}$, Bolme P, Winiarski J, Berg UB: Long-term renal function following bone marrow transplantation. Bone Marrow Transplant 2007;39:717-723.

-50 Frisk P, Bratteby LE, Carlson K, Lonnerholm G: Renal function after autologous bone marrow transplantation in children: a longterm prospective study. Bone Marrow Transplant 2002;29:129-136.

51 Jones DP, Spunt SL, Green D, Springate JE: Renal late effects in patients treated for cancer in childhood: a report from the Children's Oncology Group. Pediatr Blood Cancer 2008;51:724-731.

52 Arjmandi-Rafsanjani K, Hooman N, Vosoug P: Renal function in late survivors of Iranian children with cancer: single centre experience. Indian J Cancer 2008;45:154157.

53 Jetton JG, Ocku F, Dreyer ZE, Goldstein SL: Pediatric cancer survivors are at high risk for CKD. J Am Soc Nephrol 2009;20:748A. 